\section{Kleine aber wichtige Schritte}

\author{
Konzepte zur Umsetzung einer Nachhaltigen Regionalentwicklung setzen vorrangig an \\ der Produktlinie Ernährung an (1). Im Hinblick auf eine stärkere räumliche Bindung von \\ Wirtschaftsprozessen und die Schließung von Stoffkreisläufen fällt der Landwirtschaft \\ eine herausragende Rolle zu. Ihre spezifische Raumbindung und die damit einhergehende \\ Abhängigkeit und Nutzung von natürlichen Ressourcen beinhaliten vielfältige Handlungs- \\ möglichkeiten zur Operationalisierung des Leitbildes.
}

$\mathrm{E}$

Von Guido Nischwitz duktion und Nahrungsmittel (ca. 85 Prozent) werden im Rahmen eines mehrstufigen und indirekten Absatzes vermarktet. Zentrale Akteure sind hier neben der Landwirtschaft und den Endverbrauchern das produzierende Ernährungsgewerbe und die Handelsunternehmen. Ihre Gestaltungsspielräume für eine nachhaltige Regionalisierung sind von zahlreichen (räumlich) übergreifenden und individuellen Einflußgrößen abhängig. Von besonderer Relevanz sind dabei die politisch-rechtlichen und sozioökonomischen Rahmenbedingungen. In ihrer gesamten Wirkungsrichtung lassen sie sich als eindeutig hemmend charakterisieren (2).

In vielen ländlichen Regionen schreitet die Auflösung gewachsener regionaler Wirkungsbeziehungen und Wirtschaftskreisläufe voran. Im produzierenden Gewerbe führt der räumliche und sektorale Konzentrationsprozeß vielerorts $\mathrm{zu}$ einem Verlust an Weiterverarbeitungsstrukturen. Managementkonzepte zur Regionalvermarktung in den Produktlinien Milch, Fleisch und Gemüse werden mit dem Problem fehlender Verarbeitungsstätten konfrontiert. Zurückzuführen ist dieser Strukturwandel u.a. auf den anhaltenden Verdrängungswettbewerb im Rahmen eines steigenden Preis- und Kostendrucks. Darüber hinaus haben Europäische Union und Bund durch eine einseitige Förderpolitik zugunsten fernabsatzorientierter Betriebe und durch eine stetige Verschärfung von Rechtsvorschriften bewußt eine Strukturbereinigung auf Kosten kleinerer Wirtschaftsgefuige betrieben.

Im Ernährungshandwerk fuihren der Trend zur Filialisierung und der Bedarf an Produkten mit einem hohen Verarbeitungsgrad zur Zentralisierung der Warenbeschaffung und Lieferbeziehungen. Der steigende Konzentrationsgrad im deut- schen Lebensmittel-Einzelhandel und der verstärkte Strukturwandel zu Lasten kleinerer selbständiger Einzelhändler entzieht der Regionalisierung potentielle Kooperationspartner. Die Überregulierung der Agrarmärkte wirkt sich gleichfalls zu Lasten regionaler und traditioneller Erzeugungs- und Vermarktungsstrukturen aus.

\section{- Verbleibende Chancen}

Der verschärfte Wettbewerb auf den Agrar- und Lebensmittelmärkten bietet allerdings auch Chancen für eine nachhaltige Regionalisierung der Produktlinie Ernährung. In Teilen der Landwirtschaft setzt sich die Erkenntnis durch, daß der bisherige Entwicklungsgang den Erhalt einer flächendeckenden, wirtschaftlich und ökologisch tragfähigen Landbewirtschaftung bedroht. Die steigende Nachfrage nach frischen, gesundheitlich unbedenklichen und umweltverträglich

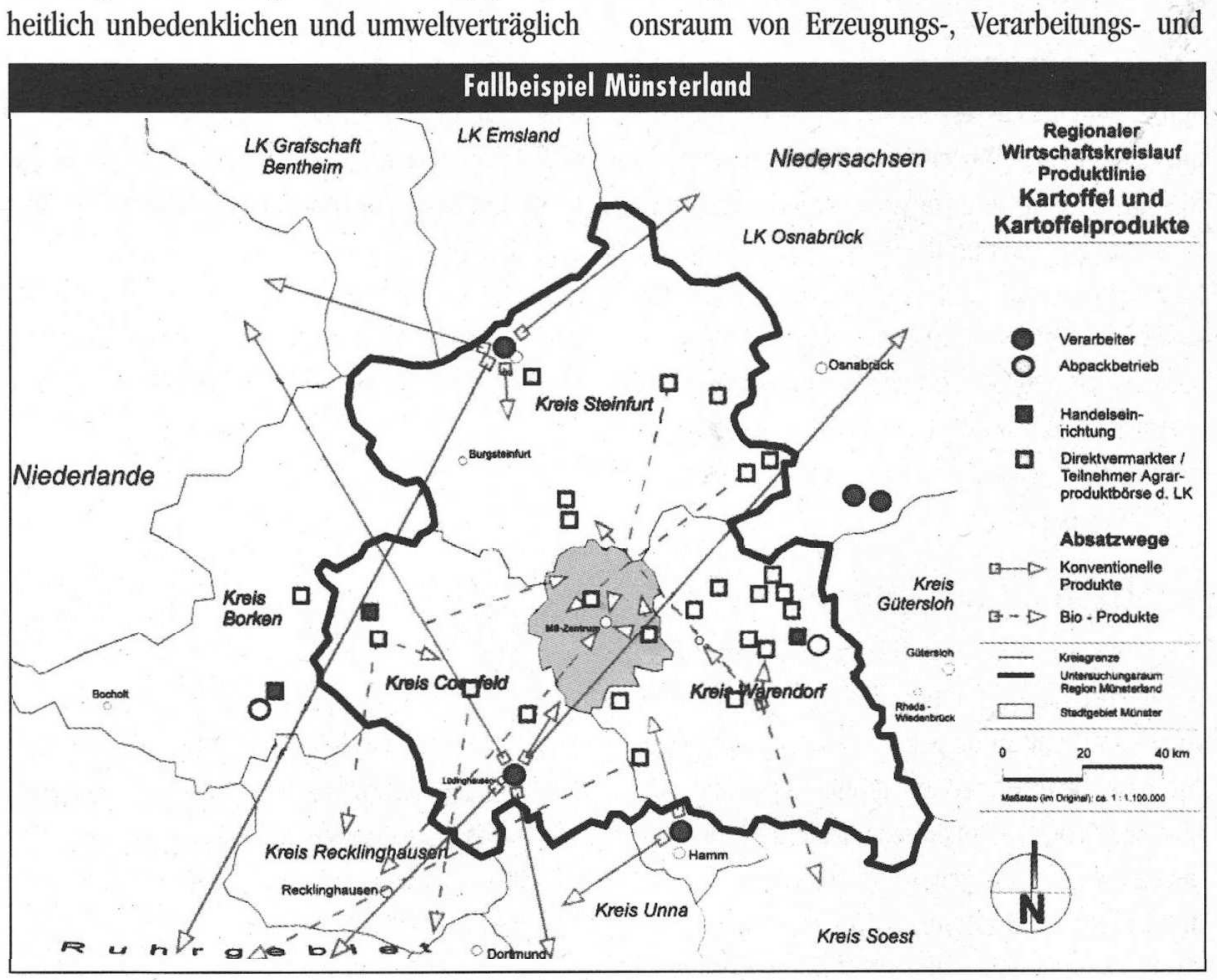

erzeugten Nahrungsmitteln weist auf eine sinnvolle Verknüpfung mit regionalen Angebotspotentialen hin (3). Die Bereitschaft, neue Wege einzuschlagen und regionale Kooperationen einzugehen, wächst zusehends. In Ernährungshandwerk und Handel ist der Bedarf an neuen Profilierungs- und Anpassungsstrategien groß. Das Angebot regional und umweltverträglich erzeugter Nahrungsmittel kann ein Mittel sein, sich von der Konkurrenz abzusetzen. Um den privaten Endverbraucher als wichtigste Zielgruppe zu erreichen, müssen allerdings mit der regionalen Herkunft produktspezifische Zusatzleistungen und Qualitätsgarantien verknüpft werden. Eine konsistente Verzahnung von Ansprüchen der Konsumenten an eine Herkunfts- und Qualitätssicherung mit Aspekten des Umwelt- und Tierschutzes kann ein erhebliches Nachfragepotential aktivieren.

\section{Das Fallbeispiel Münsterland}

Die Akteure regionaler Handlungssysteme müssen sich diesem dynamischen Spannungsfeld von fördernden und hemmenden Einflußgrößen stellen. Entscheidend ist, ob sie dabei die Ebene programmatischer Absichtserklärungen zur Regionalisierung verlassen und neue selbsttragende Strukturen initiieren können. Anhand des Modellprojekts des IÖW im Münsterland (Tabelle 1) sollen die wichtigsten Erfahrungswerte erläutert werden. Die Region Münsterland - verstanden als Akti-
onsraum von Erzeugungs-, Verarbeitungs- und 
Tabelle 1: Aktivitäten zur nachhaltigen Regionalisierung der Produktlinie Ernährung im Mönsterland

\begin{tabular}{|c|c|}
\hline Mörz 1995 & $\begin{array}{l}\text { Strategietreffen zwischen Vertretern der Stadt Münster und den Instituten für } \\
\text { Geographie der Universitöt Münster zur Förderung einer regionalen Landwirtschaft }\end{array}$ \\
\hline Juni und Dezember 1995 & $\begin{array}{l}\text { Zielfindungsgespröche der Stadt Münster: "Entwicklung einer ökologisch und } \\
\text { okonomisch orientierten Landwitschaft in der Region Münster" }\end{array}$ \\
\hline Mai 1996 & $\begin{array}{l}\text { Antrag des IÖW an die Deutsche Bundesstiftung Umwelt in Kooperation mit Umweltamt } \\
\text { und Umweltdezernat der Stadt Münster zur Förderung des Projekts: "Marktorientierte } \\
\text { Instrumente eines Iegionalen Stoffstrommanagements in der Produktlinie Ernährung" }\end{array}$ \\
\hline Juil 1996 & $\begin{array}{l}\text { Gründung des Vereins "Regionale Landwirtschaff Münsterland (RLM)", als Plattform zUr } \\
\text { Etoblierung einer umwelffreundlichen und regionalorientierten Landwirtschaft }\end{array}$ \\
\hline Winter 1996/97 & $\begin{array}{l}\text { Organisation von Aktionswochen mit Gerichten aus regionalen Produkten in der Haupt- } \\
\text { mensa der Universität und in vier städtischen Kantinen durch den RLLI }\end{array}$ \\
\hline Mai 1997 & Start des DBU. Projekts durch das IOW \\
\hline Sommer 1997 & $\begin{array}{l}\text { Analyse von Verarbeitungsstrukturen in der Produktlinie Ernährung und regionaler Nach- } \\
\text { frage durch das löW }\end{array}$ \\
\hline Dezember 1997 & $\begin{array}{l}\text { Workshop des IOW: "Chancen und Grenzen der Regionolisierung der Produktlinie } \\
\text { Ernchrung in Münster und dem Münsterland" }\end{array}$ \\
\hline Februar 1998 & $\begin{array}{l}\text { Gründung der landwirtschaftlichen Erzeugergemeinschaft „Ökologische Höfe Münster- } \\
\text { land" in Zusammenarbeit mit dem RLM }\end{array}$ \\
\hline Mä̀z 1998 & $\begin{array}{l}\text { Workshop des :OW: Regionale Produkte im Lebensmitteleinzelhandel des } \\
\text { Münsterlandes" }\end{array}$ \\
\hline Mai 1998 & $\begin{array}{l}\text { Veranstaltung des IÖW und des RLM: "Erzeugerrichtlinien und regionale } \\
\text { Herkunftszeichen - Beispiele ous anderen Regionen" }\end{array}$ \\
\hline August 1998 & $\begin{array}{l}\text { Gesprächsunde lokaler Agendo-Arbeitskreise mir dem lOW und dem RLW: „Aufbou eines } \\
\text { regionalen Guresiegels und einer Koordinierungsstelle für Regionalvermorktung" }\end{array}$ \\
\hline September 1998 & $\begin{array}{l}\text { Veronstaltung des RLM und des IOWW zur Gründung einer Anbougemeinschaft in der } \\
\text { konventionellen Landwirtschaft und zur Entwicklung eines Kriterienkatalogs }\end{array}$ \\
\hline Okiober 1998 & $\begin{array}{l}\text { Workshop des IOW: "Regionules Herkunfis- und Ouditiotszzeichen für Nohrungsmittel in } \\
\text { Münsterlond" }\end{array}$ \\
\hline
\end{tabular}

Quelle: eigene Darstellung

Vermarktungshandlungen - ist ein traditioneller Agrarwirtschaftsraum (4). Insofern ist die Produktlinie Ernährung durch zahlreiche Akteure institutionell stark besetzt. Um so überraschender war die Erfahrung, daß es den relevanten Akteuren an einem grundlegenden Vorverständnis über die regionale Ausgangslage in der Produktlinie Ernährung mangelte. Vor diesem Hintergrund gestalteten sich die Zielfindungsgespräche sehr schwierig. Eine Verständigung über den IstZustand erfolgte erst mit einer zweieinhalbjährigen „Verspätung“ durch das IÖW-Projekt. Die hierbei vorgenommene Visualisierung von Informationen über Erzeugungs- und Vermarktungsverflechtungen (siehe Karte) und das Aufzeigen von Nachfragepotentialen (vgl. Tabelle 2 ) versetzte die Akteure erstmals in die Lage, über die Ausgangslage zu reflektieren. Auf dieser Grundlage war eine Verständigung über den regionalen Handlungsbedarf und über Schwerpunktsetzungen möglich.

Trotz dieser wichtigen Schritte und der Gründung einer organisatorischen Plattform für eine Regionalvermarktung im Münsterland verläuft der Entwicklungsprozeß sehr zeit- und mit den Bedürfnissen regionaler Strukturen die Anschubfinanzierung einer Koordinierungsstelle. Gleichzeitig fehlt es zentralen Schlïsselakteuren auf der Erzeugerseite an fachlicher Kompetenz und Risikobereitschaft. Diese schwache Marktposition wird von Kooperationspartnern aus den Bereichen Weiterverarbeitung und Vermarktung für eigene Wirtschaftsinteressen ausgenutzt. Die gerade erst gebildeten Netzwerkstrukturen werden wieder gelöst, sobald man in der Lage ist, die erfaßten Marktnischen selbst zu besetzen. Darüber hinaus fehlen im Münsterland regional ausgerichtete mittelständische Verarbeitungsstrukturen.

\section{- Perspektiven}

Trotz dieser Problemlagen beinhaltet der eingeleitete Regionalisierungsprozeß erhebliche Chancen und Perspektiven für die Verstetigung einer nachhaltigen Entwicklung. Mit dem kontinuierlichen Abgleich zentraler Fragestellungen unter Nachhaltigkeitskriterien konnten viele Beteiligte für eine nachhaltige Regionalisierung der Produktlinie Ernährung sensibilisiert werden. Das Münsterland und seine Produkte verfügen in der Bevölkerung über ein hohes Ansehen, was regionalen Produkten positive Anknüpfungspunkte eröffnet. Zahlreiche Akteure aus der regionalen Großhandelsstufe, dem Einzelhandel und der Gastronomie haben dies erkannt und engagieren sich. Mit der Einrichtung einer Dachorganisation, die von zentralen Akteuren getragen wird, besteht die Basis für eine ausbaufähige Netzwerkstruktur. Der künftige Erfolg wird aber davon abhängen, inwieweit es gelingt, die regionalen Handlungsstrukturen $\mathrm{zu}$ stärken und ein wirtschaftlich tragfähiges Kooperationsmanagement zu etablieren. Hierfür müssen verstärkt langfristige Zielsetzungen und Strategien

\begin{tabular}{|l|l|c|}
\hline \multicolumn{2}{|c|}{ Tabelle 2: Nachfragepotential für Frischgemïse im Mönsterland I) } \\
\hline & Bio-Produkte & \multicolumn{1}{|c|}{ insgesamt } \\
\hline $\begin{array}{l}\text { Käuferreichweite } \\
\text { - prozentud (Alte Bundesländer)2) } \\
\text { - absolut Münsterlond }\end{array}$ & $\begin{array}{l}41,0 \text { Prozent } \\
66.135 \text { Personen }\end{array}$ & 97,3 Prozent \\
923.239 Personen
\end{tabular}


formuliert werden, um allen Beteiligten eine ökonomische Perspektive zur Umsetzung der Entwicklungskorridore aufzuzeigen.

Für eine nachhaltige Regionalisierung der Produktlinie Ernährung zeigt das Modellprojekt im Münsterland neue Handlungsfelder und Wege auf. Sie müssen nur konsequent genutzt werden.

\section{Anmerkungen}

(1) Linckh, G. et al.: Nachhaltige Land- und Forstwirtschaft. Heidelberg 1997.

(2) Nischwitz, G.: Fördernde und hemmende Faktoren für regionale Produktion und Vermarktung. Herausgegeben von Nabu und DLV, Bonn 1998.

(3) Nischwitz, G., R. Molitor: Regionale Nachfragepotentiale in der Produktlinie Ernährung. Schriftenreihe des IÖW (im Erschei nen). Berlin 1998.

(4) Fiebig, Th.: Analyse der Verarbeitungsstrukturen in der Produktlinie Ernährung und Identifikation regionaler Produktlinien im Münsterland (unveröffentlichte Studie). Wuppertal 1997.

\section{Der Autor}

Dr. Guido Nischwitz is wissensdioflither Mitarbeiter an löW im Forschungsfeld Regionde Wirtschaftspolitik/ Nachhaltige Regionalentwicklung.

Kontakt: IÖW-Regionalbüro Nordrhein-Westfalen, Völklinger Str. 9, 42285 Wupperial, Tel. 0202/80530, Fax 0202/ 83402, E-mail: mailbox@ioew.w.eunet.de

\section{Die ökonomische Säule der Nachbaltigkeit}

ist das Thema des Informationsdienstes
Ökologisches
Wirtschaften 6/98

\section{Ökologie und Wett- bewerbsfäbigkeit von Unternehmen}

ist das Thema des Informationsdienstes

\section{Ökologisches \\ Wirtschaften $1 / 99$}

Wenn Sie potentielle Beiträge haben, wenden Sie sich bitte an die Redaktion!

Pilotprojekt im Oberbergischen Kreis

\section{Wie entsteht eine „Regionale Agenda"?}

\section{Der landesweif erste Versuch, eine "Regionale Agenda" zu etablieren, findef seif April im Oberbergischen Kreis in Nordrhein-Westfalen statt. In einem zehnmonatigen Prozeß arbeiten die Kreisverwaltung und das Institut für öko- logische Wirtschaftsforschung an der Entwicklung nachhaltiger und zukunfts- weisender Projekte und Vorhaben.}

$\mathrm{D}$ er Oberbergische Kreis mit seiner Kreisstadt Gummersbach liegt im Bergischen Land, einer durch seine Mittelgebirge geprägten Kulturlandschaft. Besondere wirtschaftliche Bedeutung haben die Metallwirtschaft, der Anlagenbau und die Kunststoffverarbeitung, sowie die Landwirtschaft mit einem hohen Anteil an Dauergrünland und der Tourismus.

Die im März 1998 vom Kreis beschlossene Durchfiihrung einer ,Regionalen Agenda" wird als Pilotprojekt vom Ministerium für Umwelt, Raumordnung und Landwirtschaft des Landes Nordrhein-Westfalen gefördert.

Kerngedanke beim Vorgehen des IÖW, das mit der Durchfiuhrung beauftragt wurde, ist es,

von Anfang an in drei Handlungsfeldern zu arbeiten, die in besonderer Weise regionale Potentiale und Standortvorteile enthalten (Zukunft der Arbeit und ökologische Innovation, Zukünftiges Bauen und Wohnen, Zukunft der Landwirtschaft und des Ernährungsgewerbes, vgl. Abbildung 1),

in diesen Handlungsfeldern Akteure anzusprechen und für eine Zusammenarbeit im Agendaprozeß zu gewinnen und

bei der Integration von ökologischen, sozialen und ökonomischen Aspekten grundsätzlich auf die Erschließung von neuen Möglichkeiten der Wertschöpfung in der Region zu achten. Insbesondere durch die Zusammenarbeit mit den beruflich im jeweiligen Handlungsfeld aktiven Akteuren, den „Profis“, unterscheidet sich die „Regionale Agenda“ von den lokalen Agenden, die auf eine breite Bürgerbeteiligung setzen. Dem IÖW und dem Oberbergischen Kreis war es an dieser Stelle wichtig, im Zusammenspiel mit den Gemeinden eine Arbeitsteilung vorzunehmen.

Alle drei Handlungsfelder enthalten in besonderer Weise Potentiale und Standortvorteile des Oberbergischen Kreises und sind zugleich wichtige Handlungsfelder einer Nachhaltigen Entwicklung. Im Bereich Arbeit und ökologische Innovation geht es um Möglichkeiten einer regional bezogenen Unternehmensfuihrung, die z.B. im Kunststoffrecycling oder bei der Nutzung von Holz als regionalem Energieträger Bedeutung gewinnen kann. Das Handlungsfeld Bauen und Wohnen ist eines der klassischen Schlüsselfelder für eine Nachhaltige Entwicklung. Hier steht die Vernetzung bestehender Angebote von Handwerkern, Architekten und Bauherren im Mittelpunkt. Im Handlungsfeld Landwirtschaft und Ernährung wird es in Zukunft auch aus wirtschaftlichen Gründen notwendig sein, landwirtschaftliche Produktionen auf umweltverträgliche Verfahren umzustellen. Der regionale Kontext im Oberbergischen Kreis bietet vielversprechende Möglichkeiten, eine wachsende Nachfrage nach regional produzierten und gesunden Lebensmitteln zu erzeugen.

\section{- Vier Leitgedanken}

Für die Arbeitsweise in den Handlungsfeldern sind vier Stichworte von zentraler Bedeutung:

\section{Konsens}

Eine Verständigung innerhalb der querschnittsorientierten Arbeitsgruppen mit Teilnehmern aus Wirtschaft, Handwerk, Politik und Verwaltung ist nur möglich, insofern es gelingt, einander tatsächlich zuzuhören, sich auf die Handlungsbedingungen des jeweils anderen einzulassen und so eine gemeinsame Zielorientierung für eine nachhaltige Entwicklung im Kreis Oberberg zu gewinnen.

2. Prozeß

Die Prozeßorientierung der „Regionalen Agenda" bedeutet, daß die Inhalte - Projektideen, Konzepte - von den Akteuren aus der Region entwickelt werden. Das IÖW nimmt neben der Moderatorenrolle die Aufgabe der fachlichen Begleitung bei der Ideenfindung und deren Weiterentwicklung wahr. 
(c) 20I0 Authors; licensee IÖW and oekom verlag. This is an article distributed under the terms of the Creative Commons Attribution Non-Commercial No Derivates License (http://creativecommons.org/licenses/by-nc-nd/3.o/), which permits unrestricted use, distribution, and reproduction in any medium, provided the original work is properly cited. 